\title{
Splenectomy in non-traumatic diseases
}

Carlos Augusto Gomes ${ }^{1}$, Cleber Soares Junior ${ }^{1}$, Federico Coccolini ${ }^{2}$, Giulia Montori ${ }^{2}$, Alice Assumpção Soares ${ }^{3}$, Célio Pereira Junior³, Flávio Vieira Marques Filho3, Paula Rabelo Halfeld Mendonça ${ }^{3}$, and Felipe Couto Gomes ${ }^{3}$

1. Surgery Department, Hospital Maternidade Therezinha de Jesus, Faculdade de Ciências Médicas e da Saúde Juiz de Fora (SUPREMA), Brazil

2. General, Emergency and Trauma Surgery, Papa Giovanni XXIII Hospital, Bergamo, Italy

3. Internal Medicine Unit, Hospital Universitário Therezinha de Jesus, Faculdade de Ciências Médicas e da Saúde Juiz de Fora (SUPREMA), Brazil

\section{REVIEW}

Please cite this paper as: Gomes CA, Junior CS, Coccolini F, Montori G, Soares AA, Junior CP, Filho FVM, Mendonça PRH, Gomes FC. Splenectomy in non-traumatic diseases. AMJ 2018;11(5):295-304.

https://doi.org/10.21767/AMJ.2018.3386

Corresponding Author:

Dr. Carlos Augusto Gomes

Rua Senador Salgado Filho 510 / 1002

Bairro Bom Pastor - Minas Gerais - Brazil.

CEP: 36021-660

Phone: 05521 32-3218-3188

Email: caxiaogomes@gmail.com

\section{ABSTRACT}

Splenectomy represents the first choice for treatment of spontaneous splenic rupture, abscesses, cysts, tumours, and an alternative for control of hereditary, autoimmune, and myeloproliferative disorders. However, its relative indications have been reviewed for better understanding of both the primary affections and of splenectomy per se, particularly with regard to the immune system. The emergence of minimally invasive surgery, the possibility of splenic preservation, and availability of biological therapy has shown that the procedure has often been referred to as salvage therapy upon failure of other therapeutic propositions. On the other hand, patients have their general health status compromised by the underlying disease, by the use corticosteroids or biologic therapy, immunosuppressed, coagulation disorders, which contribute to the incidence of postoperative complications, such as infections, bleeding and venous thrombosis.
Therefore, this scenario favours higher morbidity and mortality rates than those of other intra-abdominal surgical procedures. Thus, this review has the primary and comprehensive objective of purpose the best moment for splenectomy, when surgeons can interfere in the natural course of the disease increasing patients' quality of life and survival. In short, it is desired that the surgeon has complete knowledge of the profound physiological changes imposed on the host. In addition, to distinguish when it is curative and mandatory from when it must be put on hold due to other non-operative treatments with similar outcomes and, lastly, when it is not recommended for not aggregating survival.

\section{Key Words}

Splenectomy, splenomegaly, laparoscopy

\section{What this review adds:}

\section{What is known about this subject?}

The indication of splenectomy in trauma patients is wellestablished; however in non-traumatic diseases it is much more controversial and current review and recommendations is scarce.

\section{What new information is offered in this review?}

This review explores the absolute and relative indications for splenectomy in different clinical sets. It also discourses on pathophysiological changes in an already compromised host.

3. What are the implications for research, policy, or practice?

Comprehensive review on the role of splenectomy in different non-traumatic settings, especially, haematological diseases in which it represents a rescue therapy because news biologic therapies. 


\section{Introduction}

The spleen has two main functions: a haematological function (red pulp), responsible for haemocatheresis and, therefore, stores iron, erythrocytes and platelets and an immunological function (white pulp), represented by the peri-arteriolar lymphoid sheath (PALS). This sheath acts in the production of antibodies and opsonins against immediate antigens. ${ }^{1,2}$ It is part of innate immunity wherein it phagocytes the antigen-antibody complex, synthesizes immunoglobulin $M$ by means of $B$ memory cells and produces properdin, opsonins and tuftsin. Therefore, it is the only organ capable of removing encapsulated bacteria. ${ }^{2}$

It's exeresis, thus, causes profound changes on the organism's homeostasis, in particular on the immune system. Nevertheless, it is the curative treatment for spontaneous rupture, cysts, abscesses, and some malignancies. It is also an option for acquired and congenital erythrocyte and autoimmune diseases, as well as idiopathic splenomegaly. ${ }^{3}$

The objective of the study was to review the clinical indications of splenectomy, to understand the pathophysiological aspects that it produces in the natural course of the diseases, and to review its indication in face of significant advances of biological and immunosuppressive therapies. Finally, the study also aims to provide the evidence that ensures the best moment in order to produce the desired effect and minimize postoperative complications. In addition, splenectomy in non-traumatic disease is nowadays a rescue therapy; its indications are more and more individualized. In addition, the time is even more complex and be quoted for each disorder.

\section{Indications of splenectomy}

The most frequent indications for splenectomy are blood disorders: immune thrombocytopenic purpura (ITP), refractory to clinical treatment, and haemolytic anaemias. Then, the list is completed by abscesses, cysts, primary solid lymphoproliferative tumours (Hodgkin's and non-Hodgkin's lymphoma), leukaemias, myelofibrosis, malaria, congestive splenomegaly, and Felty's syndrome (Table 1$)^{1-3}$

\section{Absolute indications}

\section{Splenic abscess}

Splenic abscess is rare and potentially fatal and is predominant in men. Diagnosis should be considered in cases of fever from unknown aetiology. The causes are haematogenous spread of bacteria by contiguity and traumas. Risk factors include neoplasia, hepatopathies, diabetes mellitus, splenic infart and immunosuppressive conditions. Abdominal tomography is the preferred test to identify the location and differentiate it from other splenic tumours; however the ultrasonography can be used. The microorganisms most frequently obtained from blood culture or drained abscess culture were Escherichia coli and Staphylococcus aureus, followed by Klebsiella pneumoniae. A few cases with multiple splenic abscesses caused by Salmonella typhi have also been described in the literature. ${ }^{4,5}$

The best treatment is still to be established. Non-operative therapy with antibiotics associated with percutaneous drainage (PCD) and/or splenectomy are feasible. On the other hand, splenectomy is the most efficient and definitive treatment, being the gold standard treatment. However, PCD has been indicated for uncomplicated cases or as a bridge for definitive treatment for high-risk individuals. Although a high failure rate has been observed for this procedure, it is an effective alternative to surgery in selected cases. Despite higher mortality, splenectomy is the only option upon failure of PCD, recurrent and multiloculated abscesses and/or thick septum and necrosis. ${ }^{4,5}$

\section{Splenic cysts}

The rare splenic cysts are asymptomatic and present higher incidence in females and includes several etiologies: primary cysts (epithelial, epidermoid), posttraumatic pseudocysts (hemorrhagic or serous), vascular (post-infarction, peliosis), congenital, parasitic (hydatid cyst is the most common, caused by Taenia echinococcus), and cystic neoplasia's (hemangioma, lymphangioma, lymphoma and metastasis). ${ }^{6,7}$ Most splenic cystic lesions are benign and distinguished from cystic metastasis by the absence of solid components, they are usually diagnosed by imaging tests. ${ }^{7}$

Splenectomy laparotomy has been the method of choice for the treatment of many splenic cysts. Their removal is recommended in symptomatic cases, when they are bigger than $5 \mathrm{~cm}$ or are susceptible to spontaneous rupture, due to its complications and because conservative treatment options do not result in good long-term control. Treatment is controversial, although it is recommended to preserve splenic tissue and less and less aggressive technique. However, this is technically difficult in large cysts or when they are located in the splenic hilum. In these cases, total splenectomy is recommended due to the risk of haemorrhage. In the other cases, partial splenectomy is accepted, preferably by laparoscopy, especially if the cyst is located at the spleen poles. ${ }^{6}$ 


\section{Spontaneous splenic rupture}

Spontaneous splenic rupture (SSR) is difficult to diagnose, its mortality rate is high (20 per cent). It causes haemorrhagic acute abdomen, particularly in young individuals. Its predisposing factors are: infectious diseases (30 per cent), particularly infectious mononucleosis, malaria tuberculosis, and dengue; haematological (27 per cent), leukaemias and lymphomas (11 per cent); gastrointestinal disease such as hepatic cirrhosis and pancreatitis (10 per cent); autoimmune disease, such as rheumatoid arthritis, vasculitis, especially Wegener (4 per cent) and others (15 per cent), such as degenerative conditions (amyloidosis), congenital (cysts and haemangiomas), metabolic diseases (Gaucher disease), or even pregnancy. In tropical countries with dengue epidemics, this possibility must always be present in medical guidelines. ${ }^{8,9}$

Splenectomy is the treatment of choice in most cases ( 85 per cent), where conservative treatment is established in particular cases of infection in young patients with stable hemodynamics. Hemodynamic instability makes urgent laparotomy as mandatory conduct. In tertiary centres, expert teams, and patients with stable hemodynamics, the laparoscopic approach is well established. ${ }^{9,10}$

\section{Tumours}

\section{Primary:}

Haemangioma is the most common benign neoplasm of the spleen. Most patients are asymptomatic and the haemangioma is found by chance during imaging, surgical procedures or necropsies. The treatment of the lesion is often splenectomy, due to the potential risk of primary or metastatic spleen lesion. However, small lesions $(<2 \mathrm{~cm})$ with homogeneous radiological characteristics and welldefined contours may be followed up by serial tests. ${ }^{11}$

Primary splenic angiosarcoma is considered the most common non-lymphoid malignancy. However, it is rare with predilection for male individuals around 60 years old. It presents different clinical morphological variability and presentation and low specificity in the imaging tests, which makes diagnosis very difficult. In this case, percutaneous biopsy is contraindicated due to the risk of splenic rupture. Thus, histopathological diagnosis can only be obtained after splenectomy, which represents the only curative treatment. The surgery can be performed by laparotomy or laparoscopy, as long as it preserves the oncological principles (splenic capsule integrity and removal of the operative part of the cavity). ${ }^{12,13}$

\section{Secondary:}

Splenic metastases are rare, according to different reports, it is prevalence ranged from 0.9 per cent to 1.86 per cent in cases of breast cancer, varying with the casuistry of the study. In an autopsy study of patients who died of epithelial carcinomas, splenic metastases were found in 4.4 per cent, of which 1.6 per cent was derived from colonic carcinoma. However, the incidence of solitary metastasis was not reported. Primary locations are the breasts, lungs, rectal cervix, ovaries, and melanomas. ${ }^{14,15}$ Splenic metastases usually occur in the context of disseminated diseases and, although mostly asymptomatic, if not treated they can cause complications such as splenic rupture. When single and metachronous, they are approached with splenectomy, preferably by laparoscopy. However, in cases of uncontrolled primary disease, percutaneous ablation techniques have been used. In the case of synchronic splenic metastasis, there is still no definition on the best form of treatment. ${ }^{15}$

\section{Relative indications for splenectomy}

\section{Sickle-cell disease}

Sickle cell disease (SCD) consists in a group of genetic disorders of haemoglobin. In this disease, under low oxygen tension the abnormal haemoglobin polymerises, distorting the red blood cells size. This sickling has effects, such as tissue damage and anaemia. Common complications of SCD include increased severity of infections, pain episodes, stroke, kidney failure, chest infections and lung damage (acute sickle chest syndrome). Other important complication is splenic sequestration that occurs when red blood cells become entrapped in the spleen, which enlarges, pooling and then destroying the red blood cells and can be acute or chronic. ${ }^{16}$

The initial management of acute splenic sequestration (ASS) includes red cell transfusion, being that the long-term management options are a splenectomy; a transfusion regime; or careful observation for early signs of ASS until the spleen gradually becomes non-functional (splenic atrophy). Splenectomy may be indicated for infants with recurrent ASS, and if complete, will prevent subsequent sequestrations; if partial, it may reduce recurrence of crises. However, there is a lack of scientific evidence to demonstrate that such a splenectomy actually improves survival and decreases morbidity from acute splenic sequestration. In fact, there are no trials to assess the benefits and risks of splenectomy when compared to transfusion programs in patients with sickle-cell anemia. ${ }^{16}$ 


\section{Splenic artery aneurysm}

Splenic artery aneurysm (SAA) has low prevalence; however is the third most common intra-abdominal aneurysm. Diagnosis is also accidental, by means of imaging tests. Its aetiology is not known, but the predisposing factors are: atherosclerosis, multiparity, abdominal traumas, portal hypertension. Pregnancy is reported as the major risk factor for rupture (10 per cent), although the percentage may be overestimated. $^{17,18}$

Aneurysms with a low risk of rupture may be treated conservatively but require regular imaging to ascertain progress. Treatment for SAA is indicated in symptomatic aneurysms larger than $2 \mathrm{~cm}$, with progressive increase, or those detected in pregnancy, childbearing age or following liver transplantation are at high risk of rupture and should undergo active treatment. The primary therapeutic approach should be endovascular therapy. When its preservation is impossible, as in cases of portal hypertension, multiple aneurysms, and hemodynamic instability, total splenectomy is performed. ${ }^{18}$

\section{HLA-ABO desensitization for kidney transplantation}

Sensitization against human leukocyte antigens is the most important impediment to renal transplantation. ${ }^{19}$ Primary sensitization results from exposure to HLA antigens by transfusion, pregnancy, or prior transplantation, and stipulates waiting on transplant lists. Thus, to control the antibody-mediated reaction (AMR), two desensitization protocols were designed for the preoperative period. The first consists of high doses of intravenous immunoglobulin (Ig/IV) and the second is plasmapheresis associated with high doses of cytomegalovirus hyperimmune globulin or $\mathrm{Ig} / \mathrm{IV}$. However, these protocols may fail or there may be AMR even after these measures. It is known that most reactions will respond to treatment with immunoglobulins. However, a subgroup of patients may develop severe AMR and rapid cortical necrosis. In this situation, salvage splenectomy has been recommended. ${ }^{19,20}$

Two studies evaluated the impact of splenectomy on posttransplant rejection. One of these studies concludes that laparoscopic splenectomy was effective in six of 11 cases evaluated, and when associated with the use of bortezomib, the range of success was increased for nine of 11 cases. In the same study, when the approach was compared with plasmapheresis associated with Ig/IV it was also more effective. A second study, with 24 patients with severe AMR resistant to traditional treatment, where all were subject to plasmapheresis and $\mathrm{Ig} / \mathrm{IV}$, the combined splenectomy and eculizumab therapy was used in five out of the 24 patients.
This combination had an effect promoting restoration of renal function in 100 per cent of cases. One possible explanation would be that splenectomy removes large amounts of active plasma cells from patients. Meanwhile, plasmapheresis performs gradual removal, and eculizumab protects the renal endothelium from the reaction mediated by the remaining antibodies. ${ }^{19,20}$ Although there are no defined protocols, up-to-date literature suggests that in cases of severe AMR, a multifactorial approach is necessary, and splenectomy is an important salvage therapy. ${ }^{20}$

\section{Erythrocyte disorders}

\section{Hereditary spherocytosis:}

Hereditary spherocytosis (HS) is the most frequent inherited red cell membrane disorder and cause of haemolysis and non-immune haemolytic anaemia. It is a disease that results from quantitative and/or qualitative changes in the erythrocyte membrane proteins that produces spherocyte with pale centre aspect in the peripheral smear examination. The clinical features are pallor due to anaemia, jaundice due to the hyperbilirubinemia and splenomegaly. This is because the spleen is the site of sequestration and phagocytosis of the non-deformable red cell that is cause of the anaemia too. ${ }^{21}$

Splenectomy should not be performed based on HS diagnosis, but only on the basis of severity of anaemia and laparoscopic surgical approach is Grecommended. Thus, it is indicated for patients diagnosed with severe HS and in anaemia patients with growth deficiency and other signs of gravity. Patients with the moderate and/or compensated disease should be individually assessed for the potential benefit of splenectomy. In children, partial splenectomy, in addition to improving anaemia, preserves the immune function of the spleen. In any case, the procedure should be considered only after 6 years of age. ${ }^{21}$

\section{Hereditary elliptocytosis:}

The hereditary elliptocytosis (HE) syndrome comprises a heterogeneous group of abnormalities characterized by mutation in genes encoding membrane or skeletal proteins, which alters membrane function and reduces red cell deformability, causing presence of erythrocytes in elliptical format during peripheral blood smear analysis. Clinical manifestations range from asymptomatic to severe transfusion-dependent haemolytic anaemia. ${ }^{21}$

Surgical treatment is rarely necessary for patients with typical elliptocytosis, it should be considered only for severe forms of HE and after five years of age. In severe cases and in cases of hereditary pyropoikilocytosis (mutation and 
spectrin deficiency), red cell transfusions may be necessary, in these situations, the surgery reduces but does not eliminate haemolysis completely. Splenectomy has proven to be curative in severe cases and, therefore, its indication is the same as that for $\mathrm{HE}^{21}$

\section{Thalassemic syndrome:}

Thalassemia syndromes are autosomal recessive disorders in which the synthesis rates of one or more globin chains are reduced, resulting in defective erythropoiesis, haemolysis, and consequent hypersplenism, increased transfusion requirements and severe accumulation of iron in the host. The severity of clinical course distinguishes two main subtypes: thalassemia major (TM) and thalassemia intermedia (TI). ${ }^{22}$ The disease courses with hypercoagulable state, thrombosis and some factors are involved. These factors include: platelet activation, pathology and alteration in red blood cells, endothelial cell and peripheral blood activation, The risk of thromboembolic complications ( 5 to 37 per cent) and their sequelae (limb lymphedema and pulmonary hypertension) may occur in short/medium intervals ( 8 years) and represents serious complications. Thus, surgical indication has been postponed or avoided, even if the alternative implies a greater need for red blood cell transfusion and iron chelation. ${ }^{22-24}$

\section{Autoimmune Haemolytic Anaemia (AIHA):}

Autoimmune haemolytic anaemia is a disorder caused by autoantibodies directed against red blood cells. The majority of cases are idiopathic; however it can be secondary to lymphoproliferative syndromes and autoimmune diseases. Diagnosis is simple; it is based on the presence of anaemia, haemolysis and anti-erythrocyte antibodies (detected by direct antiglobulin test). Treatment remains controversial in view of a low number of prospective randomized trials. Nevertheless, it is a consensus that corticosteroids represent the first line of treatment. Response must take place during the second week, while its absence by the third week represents therapeutic failure. $^{25,26}$

\section{Platelet Disorders: Idiopathic Thrombocytopenic Purpura} Idiopathic thrombocytopenic purpura (ITP) is characterized by thrombocytopenia and microangiopathic haemolytic anaemia and has no specific cause (the presence of both is necessary for diagnosis). It may cause fever and neurological deficit. Diagnosis is based on the definite presence of schizocytes in the peripheral blood smear film. Helicobacter pylori (HP) may colonize gastric mucus in children and produce inflammatory mediators and antibodies that cross-react to glycoproteins, which function as antigens and are present on the surface of platelets and blood and bone marrow megakaryocytes. Thus, its research and treatment in children with ITP has been recommended. Tests such as the urea breath test (UBT) and stool antigen are useful. Another option would be the blood antibody test (Abs), but due to the lack of specificity, this has been of little value. $^{27,28}$

First line treatment in ITP consists of the use of corticosteroids and rituximab, and for refractory patients, second line consists of immunosuppressive therapy (cyclosporine, cyclophosphamide or vincristine), or splenectomy. Laparoscopic splenectomy is preferred because of chronic steroid use, increased risk of infection, and problems with surgical site healing. Splenectomy per se may contribute to increase platelet count, but it does not eliminate ITP as antibody-coated platelets remain in circulation. Therefore, splenectomy in ITP is not curative and the disease may recur in the long term with the return of thrombocytopenia. In these cases, investigation of accessory spleen, unnoticed during the first surgical intervention, is mandatory, and its surgical removal may be necessary. ${ }^{28,29}$

\section{Hypersplenism}

These are among the main causes of spleen enlargement (splenomegaly): 33 per cent of liver diseases (cirrhosis); haematological tumours (lymphoma); infections (AIDS and endocarditis); congestive or inflammatory processes (congestive); primary disease (venous thrombosis); other causes, or unknown. Symptoms include pain in the upper left quadrant referred to the shoulder and sensation of early satiety. ${ }^{29}$ Hypersplenism (increased splenic function) is a common manifestation of portal hypertension in patients with hepatic cirrhosis, suggesting advanced disease state, and an increased risk of complications. ${ }^{29}$

The combination of thrombosis, splenomegaly, and thrombocytopenia (TST) is challenging, because anticoagulants are indicated but thrombocytopenia may increase the life threatening bleeding risk. Splenectomy could alleviate thrombocytopenia and reduce portal pressure, but it can cause post-operative thrombosis and opportunistic infections. Therefore, the rationale selective splenic artery embolization (SSAE) could be a safe and effective alternative to splenectomy for TST patients; nevertheless, few documentations exist about this approach in cirrhotic patients. ${ }^{30}$

Gastric varices (GV) are one of the most common complications for patients with portal hypertension. In the 
majority of cases endoscopic scleroterapy is the option of first choice with significant rate of success. However, in cases of left-sided, sinistral, or regional portal hypertension in patients with persistent bleeding, SSAE combined with short gastric vein proved to be an effective emergency therapeutic in few cases reports. Even so, splenectomy persists as a rescue alternative. ${ }^{31}$

Surgical approach is indicated in cases of severe thrombocytopenia associated with spontaneous bleeding, post-transplant splenic sequestration, or abdominal pain due to repeated splenic infarction. ${ }^{30}$ In these cases, splenectomy, both by laparotomy I and laparoscopy, is capable of circumventing the problem. However, the possibility of an increased risk of portal vein and splenic thrombosis, which persists despite being more pronounced in the first week, should be assessed, and studies suggest postoperative anticoagulation maintenance. ${ }^{32}$

\section{Congestive Splenomegaly}

Congestive splenomegaly (CS) is a result of persistent or chronic venous congestion of the spleen, most often caused by sinusoidal intrahepatic cirrhosis, which has progressed with portal hypertension (Banti's syndrome). Progressive liver fibrosis fosters blood diversion to the spleen and increases pressure throughout the splanchnic territory. Reactive cell hyperplasia of the red pulp endothelial reticular system also contributes to spleen enlargement and to the development of hypersplenism. Another cause of CS is splenic artery aneurysm, which can produce chronic venous obstruction by direct compression of the splenic vein. $^{1,32}$

Splenectomy is indicated for the rare patients with severe residual thrombocytopenia following porto-systemic derivation. If venous thrombosis is the underlying cause of CS, splenectomy is considered the treatment of choice. In the case of splenic artery aneurysm, the patient treated with aneurysm resection and/or complementary splenectomy has a satisfactory result. ${ }^{1,32}$

In addition to the above situations, it should be noted that, according to a recent study, the incidence of splenic vein thrombosis in patients with chronic pancreatitis revolves around 20 per cent. That said, it would be appropriated to add Splenectomy to the pancreatic procedure in patients diagnosed with portal hypertension, especially if it is accompanied by gastric varices, since this addition does not lead to an increase in morbidity or mortality. ${ }^{33}$

\section{Hematological Neoplasia's}

\section{Hodgkin and Non-Hodgkin Lymphoma:}

Splenic lymphoma may be primary or secondary. Primary splenic lymphomas (PSL) constitute a rare variety of splenic neoplasms. As a secondary lymphoid organ, the spleen is usually involved by lymphomas. ${ }^{34,35}$

While the primary originates in the organ itself, in the secondary, the splenic tissue is compromised by diffuse dissemination of Hodgkin's Lymphoma and Non-Hodgkin's Lymphoma. In imaging studies, particularly abdominal tomography, four different patterns of involvement of splenic lymphoma have been described: diffuse infiltration, where the entire spleen is affected by the tumour and manifests splenomegaly without large solid masses; small, focal infiltration or miliary nodules; infiltration with large multiple confluent nodular lesions and with bulky solid masses. $^{7}$

In cases of symptomatic splenomegaly and hypersplenism, stage of disease, patient's performance status, patients refractory to polichemotherapy associated Rituximab, which represents the treatment in PSL; splenectomy may be recommended, in selected cases as a rescue therapy. It relieves symptoms and ameliorate quality of life. To date, there is insufficient evidence to support the adoption of different strategies for treat such disease. For this reason, the association of Rituximab and polychemotherapy, preceded or not by splenectomy, may be considered as the most appropriate treatment for these types of patients. ${ }^{34}$

\section{Leukaemias:}

Among haematological malignancies, lymphoid and myeloid leukaemias are prominent, both are classified as acute and chronic. Persons with these diseases may develop significant splenomegaly and then splenic sequestration, in turn, responsible for the worsening of anaemia and preexisting thrombocytopenia inherent in the underlying bone marrow failure specifically in leukaemias. ${ }^{36,37}$

Splenectomy due to haematological malignancies presents, therefore, high morbidity and mortality rates. Patients may benefit from symptomatic relief, but should be advised about the risks involved in the surgery. ${ }^{36,37}$

\section{Myelofibrosis:}

Primary myelofibrosis is a neoplasia caused by clonal proliferation of bone marrow stem cells, abnormal expression of cytokines, medullary fibrosis of varying degrees, anaemia, splenomegaly, extramedullary haematopoiesis, and general symptoms. Extramedullary 
haematopoiesis is the primary cause of organomegaly (mainly hepatosplenomegaly) present in this disease. Complications due to splenomegaly are mainly related to mechanical symptoms (pain, early satiety, diarrhoea and distension), portal hypertension, and splenic sequestration with exacerbation of previous cytopenias. ${ }^{38-40}$

The use of ruxolitinib has contributed to reduce splenectomy for treatment of myelofibrosis. However, in thrombocytopenia $(<20 \times 103 / \mathrm{uL})$ or cytopenias associated with use of ruxolitinib, splenectomy is indicated and has proven beneficial. However, in the group $(>20 \times 103 \mu \mathrm{L})$ or transfusion-dependent anaemia, splenectomy presents modest benefits, given the severity of the disease. ${ }^{40}$

\section{Felty's Syndrome}

Felty's syndrome (FS) is a challenging subtype of seropositive rheumatoid arthritis (RA) with longstanding, severe and erosive arthropathy. FS occurs in 1-3 per cent of patients with RA and is characterized by severe joint involvement, splenomegaly and neutropenia. The complete triad of erosive RA, splenomegaly and neutropenia is not considered an absolute requirement for making the diagnosis and the mere presence of RA associated with persistent neutropenia $\left(<2000 / \mathrm{mm}^{3}\right)$ is satisfactory for establishing the diagnosis. The pathophysiology of FS is multifactorial including increased neutrophil sequestration secondary to splenomegaly, peripheral destruction of neutrophils, and failure of bone marrow to produce neutrophils. Splenomegaly, that can be manifested by abdominal pain, can indicate other complications such as liver cirrhosis or other infections, like bacterial endocarditis. Abdominal pain can be manifestation splenic infarcts, on the other hand. ${ }^{41}$

Therapeutic modalities have been used like methotrexate and growth factors for the management of severe neutropenia. It should be considered the last therapeutic modality for FS patients who have severe neutropenia (< $500 / \mathrm{mm} 3$ ) and frequent infections. The prophylactic splenectomy is not recommended. Although most all patients show some improvement in neutrophil counts after splenectomy, neutropenia reoccurs in approximately 25 per cent of the patients, it does not provide a long-lasting effect. This is because cytopenias do not only result from cellular destruction by the spleen. ${ }^{41}$

\section{Schistosomiasis}

Manson's schistosomiasis is an intrahepatic pre-sinusoidal disease endemic in some Brazilian states and represents a serious public health problem. About 2-10 per cent of patients will develop the hepatosplenic form, characterized by hepatic fibrosis, portal hypertension, splenomegaly, and episodes of gastrointestinal bleeding. Rupture of oesophageal varices are the most feared complications. Esophagogastric devascularisation and splenectomy are performed to reduce venous pressure, vessel calibre and prevent rebleeding. ${ }^{42,43}$

However, the intensity of deviation of the hepatic blood flow is responsible for changes in its biochemical markers. A recent study evaluated two groups of patients with hepatosplenic schistosomiasis, one group splenectomized, and the other, non-splenectomized. The results favoured the splenectomized group with regard to the following parameters: factors VII, VIII, IX, X, protein C, platelet count, prothrombin time and partial thromboplastin activation time. In addition, it showed reduced ALT, FA, $\gamma-G T$, and bilirubin enzymes. $^{42}$

\section{Malaria}

Malaria is an infectious febrile disease caused by protozoa of the genus Plasmodium spp. (falciparum, vivax, malariae, ovale and knowlesi). It is importance in Brazil stems from its high incidence in the Amazon Region, clinical severity, and socioeconomic losses in the at-risk population. The fundamental function of the spleen has been demonstrated in this disease, which removes cells killed or infested by parasites and returns with the intact erythrocytes into the bloodstream. Therefore, it is crucial in the control of malaria and responsible for clarification from parasites. It has also been observed that asplenic and hypoplastic individuals are more susceptible to fatal progress of the disease. Spontaneous splenic rupture due to malaria and rare cases of splenic infarction refractory to clinical treatment would be the few indications for splenectomy. ${ }^{44,45}$

\section{Complications/Laparoscopy}

The main complications of splenectomy are: haemorrhage, thromboembolism, subphrenic abscess, thoracic infection and fulminant sepsis following splenectomy (OPSI). The high rates of morbidity and mortality (30 per cent) are mainly due to haemorrhage, infections, and indication for myeloproliferative diseases, such as myelofibrosis and chronic granulocytic leukaemia. It also increases the risk of venous (espleno-portal system) or arterial thrombosis due to postoperative platelet, hence the recommendation not to neglect pre- and postoperative anticoagulation. In the thalassemic disease and sickle-cell anaemia, pulmonary hypertension has been described in a prevalence that can reach 75 per cent of cases. ${ }^{1,2,44}$ 
Bacterial infection is the most common complication of splenectomized individuals, and splenectomy leaves patients vulnerable to encapsulated germs (Streptococcus pneumoniae, Haemophylus influenzae, Neisseria meningitidis). Therefore, it is recommended that patients over two years of age and those with functional hyposplenism be vaccinated against pneumococcus, Haemophilus influenzae type $B$ and meningococcus at least two weeks before surgery. In cases of emergency splenectomy, immunization should be performed postoperatively, although the effect is not as significant. They should also undergo antibiotimicrobial chemoprophylaxis for the rest of their lives. ${ }^{1,2,44}$

The risk of overwhelming post-splenectomy infection (OPSI) is both more common and closer to the date of splenectomy. It is characterized by rapid evolution, hypotension, altered consciousness, or cardio-circulatory shock. Laparoscopic splenectomy (LS) is recommended whenever possible for the follow reasons: results in less pain, shorter hospital stay, less morbidity and mortality, earlier return to job, earlier start to diet, less wound infection and better cosmetsis. Indeed, the indications for LS are well similar to those for open procedure, being especially used in hematologic diseases such as haemolytic anaemia, haemoglobinopathies and ITP. These last patients generally use corticosteroids predisposing patients to wound infection and dehiscence. Because of this, LS is the gold standard for splenectomy for normal-sized spleens and elective splenectomies performed. Splenomegaly is a contraindication for LS in several centres, due in difficulties in controlling of spleen and technical insufficiency. A conversion rate of 6.4 per cent has been reported mainly due to the degree of splenomegaly. ${ }^{44,45}$

\section{Conclusion}

Splenectomy, despite being indicated in several conditions, is still controversial and remains an open field for new studies, particularly in its relative indications. The procedure is curative alternative in a small number of diseases, although it is a complementary treatment procedure in several other clinical conditions. However, the purpose it serves, and the change imposed on the host's homeostasis is less well known to surgeons. Unlike its indication in trauma, the patient often presents with general condition compromised by chronic disease, using corticosteroids or biological therapy, which contributes to the reduction of his immune status. Clotting disorders, changes in the number and function of platelets, and associated diseases are common. This scenario exposes individuals to serious risks and complications, with morbidity and mortality rates higher than other intra-abdominal surgical procedures.

Esplenectomy is a great therapeutic resource with benefits and potential risks that have to be weighted before surgery. The possible complications of this procedure include haemorrhage, acute pancreatitis, pulmonary atelectasis and pneumonia in an immediate scenario and post-splenectomy infection and venous thrombosis as long-term risks. Therefore, splenectomy is a serious operation which should be undertaken only after the depletion of clinic and noninvasive therapeutics.

Significant progress has been observed in the field of biological therapy for treatment of its main clinical indications, haematological conditions (immune thrombocytopenic purpura and haemolytic anaemias). It also presents increasing tendency to be used as a salvage alternative in cases refractory to other therapies. Therefore, the indication of splenectomy in non-traumatic diseases is a complex and multifactorial decision. The decision should focus on multidisciplinary and involve experienced professionals with in-depth knowledge about the consequences of this intervention on the host.

\section{References}

1. Weledji EP. Benefits and risks of splenectomy. Int J of Surg. 2014;12(2):113-9.

2. Rodeghiero F, Ruggeri M. Short and long-term risks of splenectomy for benign haematological disorders: should we revisit the indications? $\mathrm{Br} J$ Haematol. 2012;158:16-29.

3. Browning MG, Bullen $\mathrm{N}$, Nokes $\mathrm{T}$, et al. The envolving indications for splenectomy. $\mathrm{Br} J$ Haematol. 2017;177:321-4.

4. Sreekar H, Saraf V, Pangi AC, et al. A retrospective study of 75 cases of splenic abscess. Indian J Surg. 2011;73:398-402.

5. Schlittler LA, Dallagasperina VW. Cistos esplênicos nãoparasitários. Rev Col Bras Cir. 2010;37:442-6.

6. Kaza RK, Azar S, Al-Hawary MM, et al. Primary and secondary neoplasms of the spleen. Cancer Imaging. 2010;10:173-82.

7. Silva WT, Gunasekera M. Spontaneous splenic rupture during the recovery phase of dengue fever. BMC Res Notes. 2015;8:1-4.

8. Rebzulli P, Hostettler A, Schoepfer AM, et al. Systematic review of atraumatic splenic rupture. $\mathrm{Br} J$ Surg. 2009;96:1114-21.

9. Thapar PM, Philip R, Masurkar VG, et al. Laparoscopic splenectomy for spontaneous rupture of the spleen. J 
Minim Access Surg. 2016;12:75-8.

10. Pérez-Chaves G, Escárcega RO, Salazar-Ibarguen J, et al. Hemangioma esplénico. Gac Méd Méx. 2007;143:523-4.

11. Despoina M, Dionysios D, Georgios A, et al. Primary Angiosarcoma of the Spleen: An Oncological Enigma. Case Rep Oncol Med. 2014;2014:193036.

12. Ferreira BP, Rodler ET, Loggers ET, et al. Systemic therapy in primary angiosarcoma of the spleen. Rare Tumors. 2012;4:178-80.

13. Genc V, Akbari M, Karaca AS, et al. Why is isolated spleen metastasis a rare entity? Turk J Gastroenterol. 2010;21:452-3.

14. Monclova JL, Sloer ET, Solis YP, et al. Laparoscopic Approach for Isolated Splenic Metastasis: Comprehensive Literature Review and Report of 6 Cases. Surg Laparosc Endosc Percutan Tech. 2013;23:21-4.

15. Owusu-Ofuri $S$, Hirst $C$. Splenectomy versus conservative management for acute sequestration crisis in people with sickle cell disease. Cochrane Database Syst Rev. 2013;31(5):CD003425.

16. Moura R, Sobreira ML, Jaldin RG, et al. Aneurisma sacular de artéria esplênica: tratamento endovascular ou cirúrgico convencional? J Vasc Bras. 2013;12:230-3.

17. Al-Habbal Y, Christophi C, Muralidharan V. Aneurysms of the splenic artery - a review. Surgeon. 2010;8:223-31.

18. Orandi BJ, Zachary AA, Dagher NN, et al. Eculizumab and splenectomy as salvage therapy for severe antibodymediated rejection after HLA-incompatible kidney transplantation. Transplantation. 2014;98:857-63.

19. Tzvetanov I, Spaggiari $M$, Je on $H$, et al. The role of splenectomy in the setting of refractory humoral rejection after kidney transplantation. Transplantation Proc. 2012;44:1254-8.

20. Da Costa L, Galimand J, Fenneteau O, et al. Hereditary spherocytosis, elliptocytosis, and other red cell membrane disorders. Blood Rev. 2013;27:167-78.

21. Bolton-Maggs PH, Langer JC, Iolascon A, et al. Guidelines for the diagnosis and management of hereditary spherocytosis--2011 update. $\mathrm{Br} J$ Haematol. 2012;156:37-49.

22. Weatherall DJ. The inherited diseases of hemoglobin are an emerging global health burden. Blood. 2010;115:4331-6.

23. Crary SE, Buchanan GR. Vascular complications following splenectomy for hematologic disorders. Blood. 2009;114:2861-8.

24. Valent $P$, Lechner $K$. Diagnosis and treatment of autoimmune haemolytic anaemias in adults: a clinical review. Wien Klin Wochenschr. 2008;120:136-51.

25. Birgens $H$, Frederiksen $H$, Hasselbalch $\mathrm{HC}$, et al. A phase III randomized trial comparing glucocorticoid monotherapy versus glucocorticoid and rituximab in patients with autoimmune haemolytic anaemia. $\mathrm{Br} \mathrm{J}$ Haematol. 2013;163:393-9.

26. Scully M, Goodship T. How I treat thrombotic thrombocytopenic purpura and atypical haemolyticuraemic syndrome. $\mathrm{Br} J$ Haematol. 2014;164:759-66.

27. Abdollahi A, Shoar S, Ghasemi S, et al. Is helicobacter pylori infection a risk factor for idiopathic thrombocytopenic purpura in children? Ann Afr Med. 2015;14:177-81.

28. Sayani FA, Abrams CS. How I treat refractory thrombotic thrombocytopenic purpura. Blood. 2015;125:3860-7.

29. Booyer TD, Habib S. Big spleens and hypersplenism: fix it or forget it? Liver Int. 2015;35:1492-8.

30. Gangireddy VG, Kanneganti PC, Sridhar S, et al. Management of thrombocytopenia in advanced liver disease. Can J Gastroenterol Hepatol. 2014;28(10):55864.

31. Viveros PV, Monroy RH, Alva JAV. Splenomegaly. Rev Fac Med. 2013;56:37-45

32. Ingle SB, Hinge Ingle CR. Primary splenic lymphoma: Current diagnostic trends. World J Clin Cases. 2016 Dec 16;4(12):385-389.

33. Kattepur AK, Rohith S, Shivaswamy BS, et. al. Primary Splenic Lymphoma: A Case Report. Indian J of Surg Oncol. 2013;4:287-90.

34. Bickenbach KA, Gonen M, Labow DM, et al. Indications for and efficacy of splenectomy for haematological disorders. Br J Surg. 2013;100:794-800.

35. Rialon KL, Speicher PJ, Ceppa EP, et al. Outcomes following splenectomy in patients with myeloid neoplasms. J Surg Oncol. 2015;111:389-95.

36. Tefferi A. Primary myelofibrosis: 2013 update on diagnosis risk-stratification, and management. Am J Hematol. 2013;88:141-50.

37. Tefferi A. Pathogenesis of myelofibrosis with myeloid metaplasia. J Clin Oncol. 2005;23:8520-30.

38. Aruch D, Schwartz M, Mascarenhas J, et al. The continued role of splenectomy in the management of patients with myelofibrosis. Clin Lymphoma Myeloma Leuk. 2016;16:133-7.

39. Owlia MB, Newman K, Akhtari M. Felty's Syndrome, Insights and Updates. Open Rheumatol J. 2014;8:129136.

40. Leite LA, Pimenta Filho AA, Ferreira RCS, et al. Splenectomy improves hemostatic and liver functions in hepatosplenic schistosomiasis mansoni. PLoS One. 2015;10:1-10.

41. Silva-Neto WB, Quireze-Júnior C, Tredicci TM. Late results of esophagogastric devascularization and 
esplenectomy associated with endoscopic treatment in patients with schistosomiasis. Arq Bras Cir Dig. 2015;28:197-9.

42. Hwang JH, Lee CS. Malaria-Induced Splenic Infarction. Am J Trop Med Hyg. 2014;91:1094-100.

43. Waweru P, Macleod J, Gikonyo A. Complicated malaria and a covert ruptured spleen: a case report. J Surg Case Rep. 2014;2014:1-2.

44. Browning MG, Bullen $N$, Nokes $T$, et al. The envolving indications for splenectomy. $\mathrm{Br} J$ Haematol. 2017;177:321-4.

45. Bulus $H$, Mahmoud $H$, Altum $H$, et al. Outcomes of laparoscopic versus open splenectomy. J Korean Surg Soc. 2013;84:38-42.

\section{PEER REVIEW}

Not commissioned. Externally peer reviewed. OK

\section{CONFLICTS OF INTEREST}

The authors declare that they have no competing interests.

\section{FUNDING}

None

\section{ETHICS COMMITTEE APPROVAL}

The research has involved human data and has been performing in accordance with the Helsinki Declaration of 1975, as revised in 2008. It was also subjected to evaluation of the Research and Ethics Committee of Monte Sinai Hospital in Juiz de Fora, Minas Gerais, Brazil, and was approved by meeting made in September 2017. Moreover, the manuscript has not been previously published and is not under consideration in the same or substantially similar form in any other peer-reviewed media. All authors listed have contributed sufficiently to the project to be included as authors, and all those who are qualified to be authors are listed in the author byline. To the best of our knowledge, no conflict of interest, financial or other, exists.
Table 1: Absolute and relative indication of splenectomy

\begin{tabular}{|c|c|}
\hline Absolute Indications & Relative Indications \\
\hline Abscesses & Sickle-Cell Anaemia \\
\hline Cysts & Splenic Artery Aneurysm \\
\hline $\begin{array}{l}\text { Spontaneous Splenic } \\
\text { Rupture }\end{array}$ & $\begin{array}{l}\text { HLA-ABO Desensitization for } \\
\text { Kidney Transplantation }\end{array}$ \\
\hline $\begin{array}{l}\text { Tumours - primary, } \\
\text { adjacent, and metastasis }\end{array}$ & \multirow{2}{*}{$\begin{array}{l}\text { Erythrocyte Disorders } \\
\text { Hereditary Spherocytosis } \\
\text { Hereditary Elliptocytosis } \\
\text { AIHA }\end{array}$} \\
\hline & \\
\hline & Platelet Disorders: ITP \\
\hline & $\begin{array}{l}\text { Lymphoproliferative } \\
\text { Diseases: Hodgkin and Non- } \\
\text { Hodgkin } \\
\text { Lymphomas }\end{array}$ \\
\hline & Congestive Splenomegaly \\
\hline & $\begin{array}{l}\text { Splenomegaly of Unknown } \\
\text { Cause Diagnostic } \\
\text { Splenectomy }\end{array}$ \\
\hline & Schistosomiasis \\
\hline & $\begin{array}{l}\text { Hematological Neoplasia } \\
\text { Leukaemias } \\
\text { Myelofibrosis }\end{array}$ \\
\hline & Malaria \\
\hline & Felty's Syndrome \\
\hline & Thalassemic Syndrome \\
\hline
\end{tabular}

Note: Table summarizing the main absolute and relative indications of splenectomy 